\title{
Una revisión de la neuroanatomía y neurofisiología del lenguaje.
}

\author{
Neuroanatomy and neurophysiology of language: A review.
}

\author{
Hans Contreras-Pulache $e^{1,2,3, a}$, Jeel Moya-Salazar ${ }^{1,4, b}$
}

\section{Estimado Editor:}

Hemos leído con interés el artículo de revisión de Nizar El Inrani publicado en la Revista de NeuroPsiquiatria (1) el cual vamos a proceder a comentar en los siguientes puntos:

Primero, la revisión posiciona un tema en la agenda de la neurología actual. El tema es el estudio de la neuromorfofisiología del lenguaje, el artículo, siguiendo el pensamiento de L. Stowe, sostiene una crítica al "modelo clásico que atribuye las funciones del lenguaje exclusivamente a las áreas de Broca y Wernicke" (ABW) (2); y es que, en efecto, donde se hable de la neurología del lenguaje se sigue una única receta cosmopolita, casi cual dogma más que ciencia, se mencionan como mínimo (y usualmente solo) dos áreas conspicuas $(\mathrm{ABW})$ y se elabora sobre ellas un flujo de flechas que dan cuenta de los distintos y supuestos procesos complejos que suceden cuando una persona escucha o cuando pronuncia palabras.

Esta forma de explicar el lenguaje surgió a mediados del siglo XX y se ha instalado hasta nuestros años, siendo el "modo clásico" o, también, la forma hegemónica actual de explicar la relación entre lenguaje y cerebro. En más de un siglo, los investigadores en este campo, no han hecho sino agregar algunas áreas adicionales a las ABW o complejizar la naturaleza de las "flechas" que interconectan las áreas consideradas.
De modo tácito, o a veces francamente explicito, se limita el estudio del lenguaje al hemisferio izquierdo. Mientras hay quienes ni siquiera se cuestionan este "modo clásico" y lo enseñan y repiten en salones de clase y en documentos de investigación, el autor se levanta y critica este modo de restringir el estudio del lenguaje en la neuromorfofisiología de nuestro tiempo. Segundo, en contra del modelo clásico, el autor pasa revista de todas las estructuras del encéfalo relacionadas con el lenguaje y deja clara una posición: explicar el lenguaje en el sistema nervioso implica considerar el aporte de ambos hemisferios cerebrales. Es decir, el hemisferio izquierdo tanto como el derecho están involucrados cuando una persona escucha, lee, o pronuncia palabras (3); dicho de otra forma: el lenguaje se asienta en una neurobiología holocerebral. Lamentablemente, el autor intenta explicar los sustratos neurobiológicos del lenguaje siguiendo los constructos neuropsicológicos (memoria, percepción, sistema límbico, etc.) de la neurología clásica que tanto critica. La crítica del autor sobre lo clásico de la neurología pareciera solo limitarse a la neuromorfofisiología, y en especial a la neuromorfofisiología del lenguaje.

Tercero, el autor concluye su revisión con lo siguiente: "urge el establecimiento de modelos neurocognitivos basados en el tipo de estructuras neuroanatómicas involucradas que nos acerquen a una mejor comprensión del lenguaje". Coincidimos con el autor en la necesidad de resaltar la situación

\footnotetext{
Grupo de Investigación GRINA, Universidad Privada Norbert Wiener. Lima, Perú.

2 Grupo de Investigación NEURON, Universidad Nacional Mayor de San Marcos. Lima, Perú.

3 Centro de Desarrollo Integral RINCONADA. Lima, Perú.

4 Universidad Peruana Cayetano Heredia. Lima, Perú.

a Medico; Magister en Salud Pública; ${ }^{b}$ Tecnólogo Médico.
} 
actual como urgente. Sin embargo, creemos que es insuficiente pedirle dichos modelos a la neurociencia cognitiva que, por otra parte, está plagada de aquello que el autor critica. Incluso hay evidencia que sostiene que las neurociencias cognitivas carecen de sustrato filosófico suficiente como para atreverse a dar respuesta de los problemas fundamentales del ser humano (4). Consideramos que se precisa del aporte de todas las neurociencias (no solo de la neurociencia cognitiva) a fin de replantear el tema del lenguaje en la neurología (tal como sugiere el autor). Es más: esta necesidad no se restringe solo al lenguaje sino que se amplia hacia otros dominios aún oscuros para la neurología actual, por ejemplo: la naturaleza de la conciencia o la explicación de las funciones psicológicas superiores.

Justamente para promover la discusión en torno a esto es que vamos a proseguir, cerrando esta comunicación, con un hecho histórico. Para el año 2003, un equipo de trabajo liderado por el Dr. Pedro Ortiz Cabanillas (1933-2011) elaboró un texto, que permanece inédito, de alrededor de unas 100 páginas explicando, desde la perspectiva de la Teoría Sociobiológica Informacional (TSI), el desarrollo del habla (5), éste libro es un resumen sintético de las propuestas que hizo Ortiz, un año antes, en su libro "Lenguaje y habla personal" donde se elabora la explicación informacional del sistema del habla, que el autor contrapone a la existencia del lenguaje (6). Ortiz hace una psiconeurología del habla; para él, el lenguaje es una estructura material que se encuentra fuera de las personas, y que está ahí codificando (encriptando) algo que antes estuvo "dentro de la cabeza". Para Ortiz, en el cerebro hay un "sistema del habla" no hay "lenguaje" (repetimos: el lenguaje se encuentra "afuera" de la persona, y tiene una estructura históricamente determinada). Por eso mismo, el libro inédito que mencionamos arriba se titula: "El uso personal del lenguaje".

Uno de las valías de esta explicación es que no es una elaboración ad hoc desde la neurociencia para explicar el lenguaje sino que es una aplicación específica de una teoría mayor, de naturaleza sociobiológica (la Teoría Sociobiológica Informacional, TSI). Por eso mismo, no solo se cuenta con una explicación del sistema del habla y al mismo tiempo del lenguaje sino también se cuenta con una explicación de la estructura (y actividad) de la sociedad y del sistema nervioso. En particular, los planteamientos informacionales, proponen que estrictamente a nivel de las áreas corticales, el (sistema del) habla (ya no el lenguaje) se puede delimitar en las áreas corticales de transición (que por tanto son la transición, histórica o mejor: filética, entre una corteza heterotípica y una corteza homotípica eulaminar). A estas estructuras cerebrales de transición se les llama, desde una morfofisiología informacional: "preconsciencia".

Dentro de la preconsciencia se encuentran las $\mathrm{ABW}$ (que son las áreas más clásicas) pero también las áreas de Luria, de Exner y de Dehaene. Si bien todas estas áreas se localizan en el hemisferio izquierdo, la preconsciencia incluye también a los complementos de estas áreas, ubicados en el hemisferio derecho. De este modo, la preconsciencia como sustrato del sistema del habla (el "lenguaje" dentro de la cabeza de una persona) integra la transición de los paleocortical a lo neocortical en ambos hemisferios cerebrales. Así, cuando la preconsciencia es el sustrato neurobiológico de la actividad de una persona (por ejemplo cuando una persona lee o cuando muestra actividad verbal) se manifiesta como una actividad transcortical (se articula con muchas otras áreas corticales) y holocerebral (se asienta en ambos hemisferios).

Los planteamientos de la TSI aplicados al lenguaje y al habla parecen encajar de modo preciso con la demanda urgente de repensar las cosas en la neurología. A 25 años del inicio formal del desarrollo teórico de la TSI, que empezó a publicarse el año 1994, con el libro "El sistema de la personalidad" (7), pareciera ser que se van dando, en las neurociencias contemporáneas, las atmósferas necesarias para replantearnos las tradicionales definiciones en neurología. El Inrani ha demostrado cabalmente que este tiempo es una realidad presente, vigente y de la más alta trascendencia.

\section{Correspondencia:}

Jeel Moya-Salazar

Jr. Pacifico 957, Urb. Sn Felipe, Lima 07, Lima 51001, Perú.

Correo electrónico: jeel.moya.s@upch.pe

Declaración de Financiamiento: Autofinanciado por los autores.

Conflictos de Interés: Los autores declaran no tener ningún conflicto de interés.

\section{REFERENCIAS BIBLIOGRÁFICAS}

1. El Imrani N. Una revisión de la neuroanatomía y neurofisiología del lenguaje. Rev Neuropsiquiatr; 81(3):196-202. DOI: https://doi.org/10.20453/rnp. v81i3.3387

2. Stowe L, Haverkort M, Zwarts F. Rethinking the neurological basis of language. Lingua. 2005; 115: 997-1042. 
Una revisión de la neuroanatomía y neurofisiología del lenguaje.

3. Ries SK, Dronkers NF, Knight RT. Choosing words: left hemisphere, right hemisphere, or both? Perspective on the lateralization of word retrieval. Ann NY Acad Scie. 2016; 1369(1):111-131.

4. Bennett MR, Hacker PMS. Philosophical Foundations of Neuroscience. New Jersey: Wiley-Blackwell; 2003.

5. Ortiz P. El uso personal del lenguaje. En: ContrerasPulache H, editor. Sin título. Lima: En prensa por Fondo editorial EDUNEURO-RINCONADA ;2003. p. 120.
6. Ortiz P. Lenguaje y habla personal. El cerebro humano como sistema semiótico. Lima: Fondo Editorial Universidad Nacional Mayor de San Marcos; 2002.

7. Ortiz P. El Sistema de la Personalidad. En: ContrerasPulache H, editor. Lima: Fondo Editorial de la Universidad de Ciencias y Humanidades; 2016. 